[REVIEW ARTICLE]

\title{
ON CHILDREN'S SEMANTIC KNOWLEDGE: ISSUES IN THE ACQUISITION OF QUANTIFIER-NEGATION INTERACTION AND OF ADDITIVE PARTICLES
}

\author{
AKIKO TERUNUMA \\ Daito Bunka University
}

Semantics in Acquisition, ed. by Veerle van Geenhoven, Springer, Dordrecht, 2006, viii+355pp.

This paper considers two topics. One is the acquisition of quantifier-negation interaction. In the book under review, Musolino discusses quantifiernegation interaction in child language based on data obtained from English and Kannada. Taking into consideration data obtained from Japanese as well, this paper points out problems with Musolino's paper and presents a new analysis. The other topic to be considered is the acquisition of additive particles. In the book under review, Nederstigt and Bergsma investigate German and Dutch additive particles and take different positions as to whether children are adult-like in the interpretation of the particles. This paper shows that data obtained from Japanese support Nederstigt's claim.*

Keywords: language acquisition, the interface between (morpho)syntax and semantics, the relative scope of a quantifier and negation, additive particles

\section{Introduction}

The book under review here, Semantics in Acquisition edited by Veerle van Geenhoven, discusses language acquisition from a semantic perspective. In the introduction of the book, Van Geenhoven notes that a semantic point of view as well as a syntactic one is a prerequisite for better understanding of natural language and its development, and illustrates how language acquisition research and semantic theories are fruitfully related to each other in recent studies. In some studies on the acquisition of universal quantification (Philip (1995), Drozd (2001)), insights in semantics are taken

* I would like to express my gratitude to Jonathan Blaine and two anonymous EL reviewers for valuable comments and stylistic suggestions. Needless to say, all remaining errors are my own.

English Linguistics 26: 1 (2009) 224-246 -224- 
into consideration. In the semantic study of bare plurals, the data obtained in language acquisition research (Krämer (2000)) can be used as a test of particular alternative accounts. The book under review is concerned with the acquisition of semantic phenomena and tries to show "how acquisition research can gain from semantic research" (a phrase from Van Geenhoven's introduction to the book).

The book consists of a concise introduction by the editor, and thirteen articles which are divided into five parts. Part I contains three articles that deal with a variety of issues in the acquisition of the mapping of form and meaning, namely the acquisition of form and meaning of functional elements, the acquisition of nominal constituents and their interpretations, and the acquisition of the meaning of embedded past tense forms. Part II contains three articles dealing with children's interpretation of sentences containing a quantifier. Part III consists of three articles that investigate the development of the knowledge of tense and aspect, and Part IV consists of two articles that investigate the knowledge of finiteness and its development. Part V contains two articles dealing with additive particles in child language.

If one looks at these works from a broader perspective, the five parts can be put into two larger groups. First, Parts I, II and V discuss the acquisition of the knowledge related to the interface between syntax and semantics. For example, investigating how English-speaking children use and interpret definite and indefinite noun phrases, Roeper's "Watching Noun Phrases Emerge: Seeking Compositionality" in Part I claims that syntactic nodes and their hierarchical relation are specified in UG, and that the principle of compositionality holds, namely each syntactic node is linked to a particular interpretation, in child grammar as well as in adult grammar. The first two articles in Part II, Meroni, Gualmini and Crain's "Everybody Knows" and Drozd and van Loosbroek's "The Effect of Context on Children's Interpretations of Universally Quantified Sentences," discuss children's errors in interpreting universally quantified sentences (Philip (1995)). Meroni et al.'s paper presents an experimental support for the Felicity account (Crain et al. (1996)), which attributes children's errors in question to a non-grammatical factor. In contrast, Drozd and van Loosbroek's paper maintains the Weak Quantification account (Drozd and van Loosbroek (1999), Drozd (2001)), trying to explain the children's errors in question by a grammatical factor. The two articles in Part V, Nederstigt's "Additive Particles and Scope Marking in Child German" and Bergsma's "(Un)stressed Ook in Child Dutch," investigate children's knowledge of 
additive particles, whose domain of application plays a crucial role in the interpretation of a sentence. Nederstigt claims that children are the same as adults in the knowledge of additive particles, whereas Bergsma claims that they are not.

Second, Parts III and IV deal with the acquisition of the knowledge related to the interface between verbal morphology and semantics. Swift's "Stage Change and Temporal Reference in Inuktitut Child Language" in Part III, for instance, considers cross-linguistic universals and variations in children's temporal expressions. In previous studies of languages such as English, Italian and Hebrew, it is observed that in child language, telic verbs and atelic verbs have different aspectual interpretations, perfective and imperfective respectively, and that children use different morphological markings for the two types of verbs (Bloom et al. (1980), Antinucci and Miller (1976), Berman (1985)). Investigating Inuktitut-speaking children's temporal expressions, Swift claims that children universally associate telic and atelic verbs with perfective and imperfective interpretations respectively, but that it depends on their target grammar whether children use a particular overt morphological marking for each type of verb. Gretsch's "Functions of Finiteness in Child Language" in Part IV considers how the interpretations of finite and infinitive forms of verbs develop in German. According to Gretsch, at the first stage of development, finite forms are associated with a "here-and-now" reference and infinitive forms with a "not-here-and-now" reference. At the second stage, past participles are acquired for past reference, and accordingly infinitive forms are used for future reference.

In language acquisition research within the framework of generative grammar, a lot of studies have been done on children's (morpho)syntactic knowledge, which is easily observable in their utterances. Children's utterances that are different from adults' in form, such as null subjects and root infinitives, are among the major topics of investigation. In contrast, children's semantic knowledge, which is not easy to access, has not been investigated as much. The book under review is of significance in that it focuses on the acquisition of various semantic phenomena and addresses problems such as (i) whether children's semantic knowledge is different from adults' and if so, how and why, (ii) whether universals across language and cross-linguistic variations are observed in the acquisition of semantic knowledge, and (iii) when and how a particular semantic knowledge develops.

Space limitations do not allow for review and discussion of all the articles contained in the book. Thus, I mainly focus on two topics concerning children's knowledge related to the syntax-semantics interface that are of 
particular interest. One is children's scope construal of negative sentences containing a quantifier, which is discussed in Musolino's paper. The other is children's interpretation of sentences containing an additive particle, which is discussed in Nederstigt's and Bergsma's papers. Section 2 deals with the former topic. Section 3 deals with the latter. Section 4 concludes the paper.

2. The Acquisition of the Relative Scope of a Quantifier and Negation

In the previous studies on the relative scope of a quantifier and negation in child language, the problems in $(1 \mathrm{a}, \mathrm{b})$ have been discussed.

(1) a. Are children different from adults in the scope construal of negative sentences containing a quantifier, and if so, how?

b. What causes the difference?

Concerning (1a), Musolino (1998) and Lidz and Musolino (2002) have reported that children, unlike adults, display a strong preference for a particular scope reading. Lidz and Musolino (2002) further claim that the children's peculiar scope reading results from the overt c-command relation between a quantifier and negation. Concerning (1b), Musolino (1998) attributes the difference between children and adults to a grammatical factor. Musolino's paper in the book under review, "Structure and Meaning in the Acquisition of Scope," follows Musolino (1998) and Lidz and Musolino (2002) with respect to the problem in (1a), but differs from Musolino (1998) with respect to the problem in (1b) in attempting to explain the difference between children and adults by a non-grammatical factor. Section 2.1 below initially focuses on children's peculiar scope reading observed in the previous studies and Musolino's (1998) grammatical account for it. Musolino's paper in the book under review is then summarized in light of the problems in $(1 \mathrm{a}, \mathrm{b})$. Section 2.2 points out acquisitional facts that remain to be explained in Musolino. Section 2.3 presents a new analysis of children's interpretation of negative sentences containing a quantifier.

\subsection{Musolino's Claims}

In adult English, according to Musolino (1998), sentences like (2) and (3) are ambiguous between the narrow scope reading and the wide scope reading of the quantifier, as indicated in the parentheses.

(2) Every horse didn't jump over the fence.

$$
(\sqrt{ } \mathrm{NEG}>\forall, \sqrt{ } \forall>\mathrm{NEG})
$$

(3) Cookie Monster didn't eat two slices of pizza.

$$
(\sqrt{ } \mathrm{NEG}>\mathrm{TWO}, \sqrt{ } \mathrm{TWO}>\mathrm{NEG})
$$


In Musolino (1998), the reading in which the relative scope of a quantifier and negation is determined by their surface position is called the isomorphic interpretation, and the reading in which it is not is called the non-isomorphic interpretation. In sentences like (2) and (3), the wide scope reading of the quantifier in subject position and the narrow scope reading of the quantifier in object position are isomorphic interpretations, while the narrow scope reading of the quantifier in subject position and the wide scope reading of the quantifier in object position are non-isomorphic interpretations.

In order to investigate English-speaking children's interpretation of sentences like (2) and (3), Musolino (1998) conducted experiments with the truth value judgment task (TVJT) methodology (Crain and Thornton (1998)). In the experiments, sentences like (2) were judged against the context where the narrow scope reading of the quantifier was true (the $\neg \mathrm{Q}$ context), while sentences like (3) were judged against the context where the wide scope reading of the quantifier was true (the $\mathrm{Q} \neg$ context). ${ }^{1}$ Twenty children and twenty adults were tested for each type of sentence. The children's ages ranged between $4 ; 0$ and $7 ; 3$ in the case of sentences like (2), and between $3 ; 11$ and $6 ; 1$ in the case of sentences like (3).

The findings of Musolino (1998) are that in given contexts, children (tended to) reject sentences like (2) and (3), while adults accepted them. The results of Musolino's (1998) experiments are summarized in Table 1.

Table 1: The results of Musolino's (1998) experiments in English

\begin{tabular}{|l|c|c|c|c|}
\hline Subject and Context & \multicolumn{2}{|c|}{ Children } & \multicolumn{2}{|c|}{ Adults } \\
Sentence pattern & $\neg \mathrm{Q}$ & $\mathrm{Q}$ & $\neg \mathrm{Q}$ & $\mathrm{Q}$ \\
\hline$[$ Subj $\forall] \ldots$ Neg (e.g. (2)) & Rejected & - & Accepted & - \\
\hline Neg ... [Obj numeral] $\quad$ (e.g. (3)) & - & Rejected & - & Accepted \\
\hline
\end{tabular}

Based on these results, Musolino (1998) claims that children assign isomorphic interpretations to negative sentences containing a quantifier.

${ }^{1}$ In the case of negative sentences containing a universal quantifier, the set of $\neg \mathrm{Q}$ contexts includes the set of $\mathrm{Q} \neg$ contexts. That is, not only the NEG $>\forall$ reading but also the $\forall>$ NEG reading is true in a subset of $\neg$ Q contexts. In the remainder of this paper, however, I use the term $\neg Q$ contexts to refer to contexts where only the narrow scope reading of the quantifier is true. 
In sentences like (2) and (3), the overt c-command relation between a quantifier and negation coincides with their linear order. Accordingly, we cannot tell from Table 1 whether the isomorphism effect results from the overt c-command relation between a quantifier and negation or their linear order. Conducting an experiment with the TVJT methodology on Kannadaspeaking children, Lidz and Musolino (2002) address this question. They examined how Kannada-speaking children would interpret sentences like (4), which are ambiguous with respect to the relative scope of a quantifier and negation in adult Kannada. ${ }^{2}$

(4) Anoop eradu kaaru toley-al-illa.

Anoop two car wash-Inf-Neg

'Anoop didn’t wash two cars.' (لVNEG > TWO, $\sqrt{ }$ TWO $>$ NEG)

According to Lidz and Musolino (2002), sentences like (4) have the structure in (5) at overt syntax.

(5)

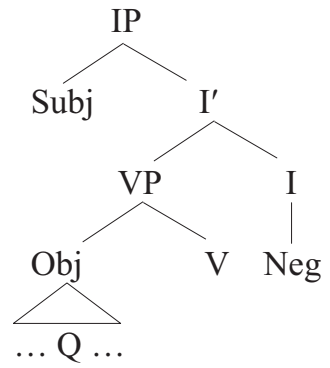

As (5) indicates, the overt c-command relation between a quantifier and negation does not coincide with their linear order in sentences like (4). The quantifier is overtly c-commanded by negation but precedes negation. Children's interpretation of sentences like (4) thus provides a good test for the question of which of the two factors, overt c-command or linear order, yields the isomorphism effect with respect to the relative scope of a quantifier and negation. In Lidz and Musolino's (2002) experiment, sentences like (4) were judged against two contexts: the $\neg \mathrm{Q}$ context and the $\mathrm{Q} \neg$ context. Twenty-four Kannada-speaking children $(4 ; 0-4 ; 11)$ and twentyfour Kannada-speaking adults were tested.

2 In this paper, I will use the following abbreviations: TTop = thematic topic particle, CTop $=$ contrastive topic particle, Acc $=$ accusative, $\mathrm{Gen}=$ genitive, $\mathrm{CL}=$ classifier, Past $=$ past tense morpheme, $\mathrm{Inf}=$ infinitive, $\mathrm{Neg}=$ negative morpheme, $\mathrm{SFP}=$ sentence-final particle. 
What Lidz and Musolino (2002) found is that children accepted sentences like (4) in $\neg \mathrm{Q}$ contexts but rejected the sentences in $\mathrm{Q} \neg$ contexts, while adults accepted the sentences in both $\neg \mathrm{Q}$ and $\mathrm{Q} \neg$ contexts. These results are summarized in Table 2.

Table 2: The results of Lidz and Musolino's (2002) experiment in Kannada

\begin{tabular}{|l|c|c|c|c|}
\hline Subject and Context & \multicolumn{2}{|c|}{ Children } & \multicolumn{2}{c|}{ Adults } \\
Sentence pattern & $\neg \mathrm{Q}$ & $\mathrm{Q}$ & $\neg \mathrm{Q}$ & $\mathrm{Q} \neg$ \\
\hline [Obj numeral] ... Neg $\quad$ (e.g. (4)) & Accepted & Rejected & Accepted & Accepted \\
\hline
\end{tabular}

Lidz and Musolino (2002) interpret these results as indicating that the isomorphism effect with respect to the relative scope of a quantifier and negation results not from their linear order but from their overt c-command relation. The results of their experiment also show that the isomorphism effect is observed in the acquisition of a language other than English.

Musolino (1998) claims that the isomorphism effect derives from the interaction of specifications in UG and the Subset Principle (cf. Crain et al. (1994)). ${ }^{3}$ Consider first sentences like (2), repeated here as (6).

(6) Every horse didn't jump over the fence.

Musolino (1998) assumes the binary parameter that determines what scope reading is allowed in negative sentences containing the universal quantifier every and its counterpart in other languages in subject position. On one value of the parameter, negative sentences in question have only isomorphic interpretations. On the other value, they can have both isomorphic and non-isomorphic interpretations. The two values are in subset/superset relation in terms of the interpretation that they allow. Following the Subset

3 Investigating the acquisition of the interpretation of sentences containing only, Crain et al. (1994) propose that the Semantic Subset Principle in (i) is operative in child grammar in order to avoid learnability problems.

(i) If the interpretative component of UG makes two interpretations, A and B, available for a sentence, $\mathrm{S}$, and if interpretation $\mathrm{A}$ makes $\mathrm{S}$ true in a narrower range of circumstances than interpretation B does, then interpretation A is hypothesized before B in the course of language development.

The Semantic Subset Principle in (i) operates when two interpretations of a sentence are in a subset/superset relation in light of contexts where they hold. As we will see in the text, Musolino (1998) applies this logic to options in UG that are in a subset/superset relation in light of interpretations that they are associated with. 
Principle, children initially set the value of the parameter to the one that allows only isomorphic interpretations.

Musolino (1998) applies a similar logic to the isomorphism effect in sentences like (3), repeated here as (7).

(7) Cookie Monster didn't eat two slices of pizza.

He assumes, essentially along the lines of Hornstein (1984), that quantifiers are classified into two types in UG. One is type I quantifiers, which are interpreted either by movement operations (May (1985), Hornstein (1995)) or by a non-movement-based interpretive mechanism (Heim (1982), Reinhart (1997)). The other is type II quantifiers, which are interpreted only by movement operations. Type II quantifiers make a subset of type I quantifiers in terms of the interpretive option. Innately endowed with the knowledge of the dichotomy of quantifiers, children learn to which of the two types each quantifier in the primary linguistic data belongs. Guided by the Subset Principle, children initially hypothesize that all quantifiers are of type II. According to Musolino (1998), it is specified in UG that while type I quantifiers allow both isomorphic and non-isomorphic interpretations with respect to the scope interaction with negation, type II quantifiers allow only isomorphic interpretations. Thus, negative sentences containing a quantifier are assigned only isomorphic interpretations in child grammar.

Musolino's paper in the book under review follows Lidz and Musolino (2002) in claiming that the isomorphism effect is found in child language with respect to the relative scope of a quantifier and negation, and that the overt c-command relation between a quantifier and negation yields the isomorphism effect. However, Musolino's (1998) grammatical account for the isomorphism effect is not adopted in Musolino's paper in the book under review, taking later experimental studies into consideration. The grammatical account predicts that children cannot access non-isomorphic interpretations. The results of Musolino and Lidz's (2006) experiment on English-speaking children demonstrate, however, that children can access non-isomorphic interpretations in negative sentences containing every in subject position when the sentences are preceded by affirmative statements as in (8).

(8) Every horse jumped over the log but every horse didn't jump over the fence.

Furthermore, the results of Musolino and Lidz's (2003) experiment on English-speaking adults indicate that in a certain experimental situation, even adults show a preference for isomorphic interpretations in negative sentences containing two in object position. Based on these findings, Musolino con- 
cludes that the isomorphism effect does not represent the difference in grammar between children and adults.

As an alternative to the grammatical account, Musolino proposes a processing account, in which the isomorphism effect is attributed to children's insufficient processing ability. Under his processing account, the isomorphism effect in sentences like (2) and (3) above is accounted for in a different manner. Consider negative sentences containing a universal quantifier such as (2), repeated here as (9), first.

(9) Every horse didn't jump over the fence.

According to Musolino, English-speaking adults prefer the NEG $>\forall$ reading in sentences like (9) because they compute as scalar implicatures (SIs) that statements like (10), which are equivalent to the $\forall>$ NEG reading, are false.

(10) None of the horses jumped over the fence.

Musolino then argues that children cannot access the NEG $>\forall$ reading of sentences like (9) because they fail to compute SIs due to their insufficient processing ability and that they consequently assign the $\forall>$ NEG reading, which is an isomorphic interpretation, to such sentences.

Next, consider negative sentences containing a numeral such as (3), repeated here as (11).

(11) Cookie Monster didn't eat two slices of pizza.

Musolino claims that in sentences like (11), children prefer isomorphic interpretations because non-isomorphic interpretations are difficult for them to access. He suggests two possible explanations of why children cannot access non-isomorphic interpretations. One possibility relates to the relative difficulty of processing isomorphic and non-isomorphic interpretations. The processing of non-isomorphic interpretations is much more difficult for children than for adults. In online comprehension of negative sentences containing a quantifier, children initially access isomorphic interpretations and cannot revise the initial parse to get non-isomorphic interpretations because of their limited computational resources. The other possibility relates to the principle of charity, which requires one to override the difficulty of processing a particular interpretation and judge the sentence true on the interpretation in a relevant context. Children do not apply the principle of charity in the way adults do and consequently do not allow non-isomorphic interpretations. 
2.2. Problems with Musolino's Claims

With respect to the problems in $(1 \mathrm{a}, \mathrm{b})$ above, Musolino's paper in the book under review makes the following claims.

(12) a. Children strongly prefer isomorphic interpretations in negative sentences containing a quantifier, and the isomorphism effect with respect to the relative scope of a quantifier and negation results from their overt c-command relation.

b. The isomorphism effect is explained in terms of processing. In the case of negative sentences containing a universal quantifier, children with insufficient processing ability fail to compute SIs, and consequently prefer isomorphic interpretations. In the case of negative sentences containing a numeral, children prefer isomorphic interpretations because they have difficulty in accessing non-isomorphic interpretations due to their insufficient processing ability.

Section 2.2.1 below shows findings from child Japanese that are incompatible with Musolino's claim in (12a), drawing on Terunuma (2008). Section 2.2.2 discusses the results of Musolino's (1998) experiment that remain to be explained under Musolino's claim in (12b). Section 2.2.3 presents a new analysis of children's interpretation of negative sentences containing a quantifier.

\subsubsection{Consideration of Musolino's Claim in (12a)}

Let us consider what prediction Musolino's claim in (12a) makes for Japanese negative sentences containing the universal quantifier zenbu 'all' or the numeral $n i$ 'two' in post-Acc position, which are exemplified in (13) and (14). In adult Japanese, these sentences are ambiguous with respect to the relative scope of a quantifier and negation.

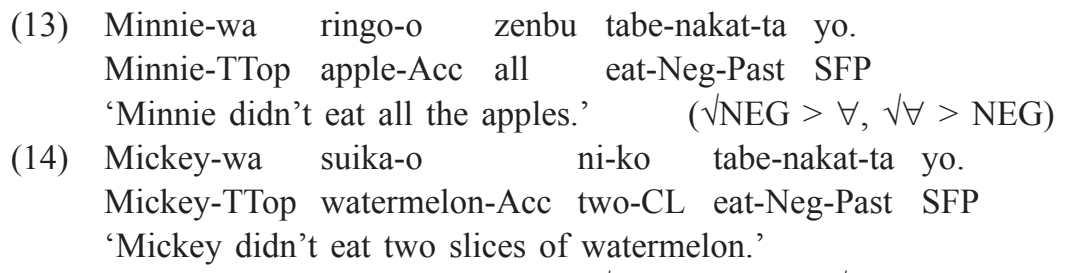

$(\sqrt{ } \mathrm{NEG}>\mathrm{TWO}, \sqrt{ } \mathrm{TWO}>\mathrm{NEG})$

Let us assume that post-Acc quantifiers are a part of object nominal projections, following Kamio (1983), Watanabe (2006) and others, and that negation affixed to $\mathrm{V}$ is in a position higher than $\mathrm{V}$ at overt syntax, as Lidz and Musolino (2002) do in the case of Kannada. Then, the structure of sen- 
tences like (13) and (14) at overt syntax is roughly (15).

(15)

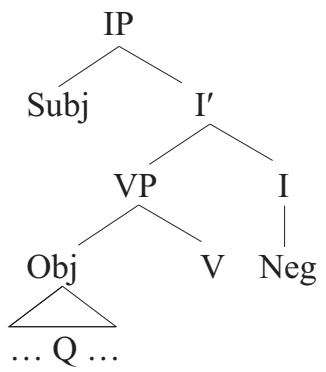

Since the quantifier is c-commanded by negation in (15), Musolino's claim predicts that Japanese-speaking children prefer the narrow scope reading of the quantifier in sentences like (13) and (14): Sentences like (13) and (14) should be accepted in $\neg \mathrm{Q}$ contexts by Japanese-speaking children.

The results of Terunuma's (2008) experiment with sentences like (13) and (14), however, show that Musolino's prediction does not hold. In the experiment, the TVJT methodology was used, and the two types of negative sentences were judged against two contexts: the $\neg \mathrm{Q}$ context and the $\mathrm{Q} \neg$ context. Table 3 summarizes the results of the experiment with respect to thirty children $(3 ; 10-5 ; 3)$ and fifteen adults who completed the test on each type of sentence.

Table 3: The results of Terunuma's (2008) experiment in Japanese

\begin{tabular}{|l|c|c|c|c|}
\hline Subject and Context & \multicolumn{2}{|c|}{ Children } & \multicolumn{2}{c|}{ Adults } \\
\hline Sentence pattern & $\neg \mathrm{Q}$ & $\mathrm{Q}$ & $\neg \mathrm{Q}$ & $\mathrm{Q}$ \\
\hline$[$ Obj $\forall] \ldots$ Neg $\quad$ (e.g. (13)) & Rejected & Accepted & Accepted & Accepted \\
\hline$[$ Obj numeral] ... Neg (e.g. (14)) & Rejected & Rejected & Accepted & Accepted \\
\hline
\end{tabular}

Japanese-speaking children rejected both types of sentences in $\neg \mathrm{Q}$ contexts, as opposed to Musolino's prediction.

2.2.2. Consideration of Musolino's Claim in (12b)

Musolino in the book under review proposes a processing account for the isomorphism effect. On his processing account, children cannot obtain the NEG $>\forall$ reading, which is a non-isomorphic interpretation, in negative sentences containing a universal quantifier such as (16) because they cannot compute as SIs that propositions equivalent to the $\forall>$ NEG reading are 
false.

(16) Every horse didn't jump over the fence.

Musolino's processing account for the isomorphism effect in sentences like (16), which contain a universal quantifier in subject position, cannot explain English-speaking children's interpretation of sentences like (17), which contain a universal quantifier in object position.

(17) The Smurf didn't buy every orange.

English-speaking children's interpretation of sentences like (17) is investigated in Musolino (1998) through an experiment on twenty children $(3 ; 11-6 ; 0)$ with the TVJT methodology. In the experiment, sentences like (17) were judged against $\neg \mathrm{Q}$ contexts. Table 4 shows the result.

Table 4: The result of Musolino's (1998) experiment in English

\begin{tabular}{|l|c|c|}
\hline \multicolumn{1}{|r|}{ Subject and Context } & \multicolumn{2}{|c|}{ Children } \\
Sentence pattern & $\longrightarrow \mathrm{Q}$ & $\mathrm{Q}-$ \\
\hline Neg ... [Obj $\forall] \quad($ e.g. (17)) & Accepted & - \\
\hline
\end{tabular}

Table 4 indicates that English-speaking children assign the NEG $>\forall$ reading to sentences like (17). In adult English, sentences like (17) are the same as sentences like (16) in that they induce SIs that propositions like (18), which are equivalent to the $\forall>$ NEG reading, are false.

(18) The Smurf didn't buy any oranges.

On Musolino's processing account, it is not clear why English-speaking children who fail to compute SIs can access the NEG $>\forall$ reading in sentences like (17), although they cannot do so in sentences like (16). The result of Musolino's (1998) experiment shown in Table 4 remains to be explained in Musolino.

So far, I have pointed out problems with Musolino's claims on the isomorphism effect observed in child language with respect to negative sentences containing a quantifier. In the next section, I would like to present a new analysis of children's interpretation of such sentences.

\subsubsection{A New Analysis}

In the experiment in Japanese, as Table 3 above shows, children's responses to negative sentences containing a quantifier differ depending on whether the quantifier is a universal quantifier or a numeral even if the quantifiers are in the same syntactic position. Based on this, I would like to consider that children interpret negative sentences containing a universal 
quantifier and those containing a numeral in different ways. More specifically, my view is that although children's acceptance/rejection of negative sentences containing a universal quantifier reflects their scope construal of such sentences, their acceptance/rejection of negative sentences containing a numeral reflects their referential interpretation of noun phrases containing a numeral. ${ }^{4}$ On this view, the isomorphism effect with respect to the relative scope of a quantifier and negation results not from their overt c-command relation but from their linear order, as opposed to Musolino's claim in (12a) above.

Let us consider how the referentiality analysis of noun phrases containing a numeral explains English-speaking and Japanese-speaking children's responses to negative sentences containing a numeral summarized in Tables 1 and 3 above. As Table 3 indicates, Japanese-speaking children reject sentences like (14), repeated here as (19), in $\neg \mathrm{Q}$ contexts.
(19) Mickey-wa suika-o ni-ko tabe-nakat-ta yo.
Mickey-TTop watermelon-Acc two-CL eat-Neg-Past SFP
'Mickey didn't eat two slices of watermelon.'

This is because they interpret sentences like (19) in a similar way as Japanese-speaking adults interpret sentences like (20), in which the object noun phrase containing a numeral is interpreted as being referential.
(20) John-wa sorera-no ni-ko-no ringo-o tabe-nakat-ta.
John-TTop those-Gen two-CL-Gen apple-Acc eat-Neg-Past
'John didn't eat those two apples.'

In adult Japanese, (20) is judged to be false in the context in (21).

(21) Of the two apples referred to, John ate one but didn't eat the other.

This means that Japanese-speaking adults reject sentences like (20) in $\neg \mathrm{Q}$ contexts. In adult Japanese, noun phases containing a post-Acc numeral such as the object noun phrase of (19) are not interpreted as referential. In child Japanese, such noun phrases are interpreted as referential. Japanesespeaking children consequently reject sentences like (19) in $\neg$ Q contexts. ${ }^{5}$

${ }^{4}$ On children's interpretation of noun phrases containing a numeral, similar discussion is found in $\mathrm{Su}$ (2001). Su (2001) claims that noun phrases containing a numeral are non-quantificational and used to refer to something specific in child Chinese.

5 As can be seen in Table 1 in the text, it is not examined in Musolino (1998) whether English-speaking children accept negative sentences containing a numeral in object position such as (i) in $\neg \mathrm{Q}$ contexts.

(i) Cookie Monster didn’t eat two slices of pizza. 
As indicated in Tables 1 and 3 above, both English-speaking children and Japanese-speaking children reject negative sentences containing a numeral in object position in $\mathrm{Q} \neg$ contexts. On the referentiality analysis, this is because $\mathrm{Q} \neg$ contexts paired with such sentences are confusing for children who interpret noun phrases containing a numeral as referential. Take a test item used in Terunuma's (2008) experiment, which is given in (22), for example.

(22) Outline of the Context Story:

An ant is going to have lunch. There are two peaches and four strawberries. He first considers eating the peaches, and eats one of them. He does not eat the other peach. Next he considers eating the strawberries. He eats two of them, but does not eat the other two.

Test sentence:

Ari-san-wa ichigo-o ni-ko tabe-nakat-ta yo.

ant-TTop strawberry-Acc two-CL eat-Neg-Past SFP

'The ant didn't eat two strawberries.'

In the context outlined in (22), the ant eats two strawberries and leaves the other two uneaten. In this situation, two pairs of strawberries are salient: a pair of strawberries eaten and a pair of strawberries uneaten. We will call the eaten pair A and the uneaten pair B. For children who interpret the object noun phrase containing $n i$ 'two' of the sentence in (22) as referential, either A or B could be the referent. This is where confusion comes from. In the given context, the sentence in (22) is false when A is chosen as the referent of the object noun phrase, but is true when B is chosen as the referent. Some children choose A as the referent, and reject the sen-

Lidz and Musolino (2002), however, investigate this by conducting an experiment on twenty-four English-speaking children $(3 ; 11-4 ; 11)$. In their experiment, children accepted sentences like (i) in $\neg \mathrm{Q}$ contexts. Under the referentiality analysis, English-speaking children's acceptance of sentences like (i) in $\neg \mathrm{Q}$ contexts is accounted for as follows: In adult English, the definite object noun phrase containing a numeral in (ii) has referential interpretation.

(ii) John didn't eat the two apples.

Three informants of mine, who are adult native speakers of English, judged that (ii) could be true in the context in (iii).

(iii) Of the two apples referred to, John ate one but didn't eat the other.

This means that English-speaking adults accept sentences like (ii) in $\neg \mathrm{Q}$ contexts. English-speaking children interpret sentences like (i) in a similar way as English-speaking adults interpret sentences like (ii) and accept them in $\neg \mathrm{Q}$ contexts. 
tence in the given context. Some other children make a guess and reject the sentence. This causes the low acceptance rate of the sentence in the $\mathrm{Q} \neg$ context. The same explanation holds for the results of Musolino's (1998) experiment on English-speaking children with negative sentences containing a numeral such as (3), repeated here as (23).

(23) Cookie Monster didn't eat two slices of pizza.

The $\mathrm{Q} \neg$ context paired with the sentence in (23) in Musolino's (1998) experiment, for example, is that Cookie Monster eats two slices of pizza and leaves the other two uneaten. Like the $\mathrm{Q} \longrightarrow$ context in (22), Q $\neg$ contexts paired with sentences like (23) in Musolino's (1998) experiment cause children confusion. ${ }^{6,7}$

Musolino in the book under review follows Lidz and Musolino (2002) in claiming that the isomorphism effect in negative sentences containing a quantifier results from the overt c-command relation between a quantifier and negation. Under the referentiality analysis above, however, his claim cannot be maintained. His claim is mostly based on the results of the experiment on Kannada-speaking children with negative sentences containing a numeral. On the referentiality analysis, children's acceptance/rejection

${ }^{6}$ Children who choose the uneaten pair as the referent of the object noun phrase of the sentences in (22) and (23) in the text and some of the children who make a guess should accept the sentences in given Q $\neg$ contexts. In the experiments in English and Japanese conducted by Musolino (1998) and Terunuma (2008), children sometimes accept negative sentences containing a numeral in $\mathrm{Q} \neg$ contexts.

7 In Lidz and Musolino's (2002) experiment, as summarized in Table 2 in the text above, negative sentences containing a numeral in object position such as (i) were accepted in $\neg \mathrm{Q}$ contexts and rejected in $\mathrm{Q} \neg$ contexts by Kannada-speaking children.

(i) Anoop eradu kaaru toley-al-illa.

Anoop two car wash-Inf-Neg

'Anoop didn't wash two cars.'

$\mathrm{Q} \neg$ contexts that were paired with sentences like (i) in Lidz and Musolino's (2002) experiment are the same in design as those that were paired with sentences like (23) in the text in Musolino's (1998) experiment. They are confusing for children who interpret noun phrases containing a numeral as referential. Accordingly, Kannada-speaking children's rejection of sentences like (i) in $\mathrm{Q} \neg$ contexts is accounted for under the referentiality analysis. Further investigation is needed, however, concerning Kannada-speaking children's response to sentences like (i) in $\neg \mathrm{Q}$ contexts. As mentioned in note 5, English-speaking adults accept negative sentences like (ii), in which the object noun phrase containing a numeral is interpreted as referential, in $\neg \mathrm{Q}$ contexts.

(ii) John didn't eat the two apples.

If Kannada-speaking adults accept Kannada sentences corresponding to (ii) in $\neg Q$ contexts, Kannada-speaking children's acceptance of sentences like (i) in $\neg$ Q contexts can be explained under the referentiality analysis. 
of negative sentences containing a numeral is not dependent on their scope construal of such sentences.

It is plausible to consider, on the other hand, that the children's acceptance/rejection of negative sentences containing a universal quantifier reflects their scope construal of the sentences. The results of the experiment on Japanese-speaking children with negative sentences containing a universal quantifier such as (13), repeated here as (24), then show that the isomorphism effect with respect to the relative scope of a quantifier and negation results from their linear order.

$$
\begin{aligned}
& \text { Minnie-wa ringo-o zenbu tabe-nakat-ta yo. } \\
& \text { Minnie-TTop apple-Acc all eat-Neg-Past } \\
& \text { 'Minnie didn't eat all the apples.' }
\end{aligned}
$$

In sentences like (24), the universal quantifier precedes negation, while it is overtly c-commanded by negation. In such sentences, as Table 3 above indicates, the quantifier, namely the element preceding negation, takes wide scope with respect to negation in child Japanese.

What remains to be considered is why the isomorphism effect is observed in negative sentences containing a universal quantifier. In the previous section, I have pointed out that Musolino's processing account that takes SIs into consideration is not tenable. However, the isomorphism effect in negative sentences containing a universal quantifier can be accounted for along the lines of another type of processing account proposed in Musolino in the book under review, which is originally proposed to explain the isomorphism effect in negative sentences containing a numeral. In negative sentences containing a universal quantifier, children with insufficient processing ability prefer isomorphic interpretations, where the element earlier in linear order takes wide scope, because the processing of non-isomorphic interpretations is difficult for children and they cannot overcome the difficulty. ${ }^{8,9}$

8 The analysis of children's interpretation of negative sentences containing a quantifier in the text above is based on Terunuma (2008). See Terunuma (2008) for more discussion. For different accounts for children's peculiar scope reading, see Gualmini (2003), Hulsey et al. (2004) and Gualmini et al. (2008).

9 The discussion on children's scope construal has been gaining a broader empirical basis in recent research. Moscati and Gualmini (to appear) experimentally investigate how English-speaking and Italian-speaking children interpret negative sentences containing not a quantifier but a modal verb. An English example of such sentences is given in (i).

(i) The lion cannot be in the same cage as the tiger.

In adult English, non-isomorphic interpretations are strongly preferred in sentences like (i). According to Moscati and Gualmini (to appear), children as well assign non-isomor- 


\section{The Acquisition of Additive Particles}

This section turns to children's knowledge of additive particles. In section 3.1, I review Nederstigt's "Additive Particles and Scope Marking in Child German" and Bergsma's "(Un)stressed Ook in Child Dutch" in the book under review. These two papers take different positions as to whether children are adult-like in the computation of a meaning contributed by additive particles. The computation of the additive meaning involves establishing a set of alternatives. Taking into consideration children's interpretation of sentences containing another type of particle that also requires a set of alternatives to be established, I show in section 3.2 that children are adultlike in the knowledge responsible for a set of alternatives and the meaning that is computed based on such a set.

\subsection{Two Different Views on Additive Particles in Child Language}

Nederstigt investigates the acquisition of two types of German additive particles: auch 'also' and noch 'still, also.' In order to interpret sentences containing an additive particle, the domain of application for the particle needs to be determined. In (25), for example, when the domain of application for auch is regarded as einen Apfel 'an apple,' the sentence has a composite meaning of (26a) and (26b).

(25) Tom hat auch einen Apfel gegessen.

Tom has also an apple eaten

'Tom also ate an apple.'

(26) a. Tom ate an apple.

b. Tom ate something other than the apple.

If auch is removed from (25), the sentence does not imply (26b). (26b) is thus a meaning contributed by the additive particle. An additive meaning like (26b) is computed based on a set of alternatives, which consists of the denotation of the domain of application for an additive particle and entities whose semantic type is the same as that of the denotation of the domain of application for the particle.

Nederstigt analyzes a longitudinal corpus of a German-speaking child to investigate the child's utterances containing auch or noch in terms of the

phic interpretations to sentences like (i). It remains to be investigated why the isomorphism effect is observed in negative sentences containing a quantifier but not in sentences like (i). 
syntactic position of the particles with respect to their domain and elements consisting of a set of alternatives, taking a close look at the context of the utterances. According to Nederstigt, the corpus analysis indicates that the child was the same as adults in the knowledge of the two additive particles before the age of 3 .

Bergsma, on the other hand, investigates the acquisition of the Dutch additive particle ook 'also, too.' In adult Dutch, according to Bergsma, the domain of application for ook varies depending on which syntactic position the particle occurs in and whether the particle receives stress or not. Consider (27)-(29).

(27) Ook [de jongen] aait de hond.

'The boy too is petting the dog.'

(28) De jongen aait ook [de hond].

'The boy is also petting the dog.'

(29) [De jongen] aait OOK de hond.

'The boy too is petting the dog.'

When ook is in presubject position as in (27), its domain is the subject noun phrase. When ook is in postverbal position and is unstressed as in (28), its domain is an element within VP. When ook is in postverbal position and is stressed as in (29), its domain is the subject noun phrase.

In order to investigate how sentences like (27)-(29) would be interpreted in child Dutch, Bergsma conducted an experiment on forty-four Dutchspeaking children $(4 ; 2-7 ; 11)$ with a picture verification task. Children were given each of the three types of sentences with three pictures and were asked to select one picture that matched a given sentence. For each of the sentences in (27)-(29), three pictures that depicted the events in (30)-(32) were shown after the four characters, namely a boy, a girl, a dog and a cat, were introduced.

(30) Both the girl and the boy are petting the dog.

(31) The boy is petting both the dog and the cat.

(32) The boy is petting the dog.

In the experiment, the younger children consistently selected the picture corresponding to (32) for all the three sentences more often than the older children. Based on this finding, Bergsma claims that children are initially insensitive to an additive meaning contributed by ook.

Nederstigt and Bergsma have come to an opposing conclusion as to children's knowledge of the meaning contribution of additive particles. Nederstigt claims, based on the production data of a child, that children can establish a set of alternatives and compute an additive meaning based on such 
a set early on. In contrast, Bergsma claims, based on the comprehension data of 4- to 7-year-old children, that children initially do not compute an additive meaning. In the next section, I show that the comprehension data obtained in Terunuma (2004) lend some support to Nederstigt's claim.

\subsection{Children's Knowledge of a Set of Alternatives}

If children are initially insensitive to the meaning contribution of additive particles, as Bergsma claims, then either of the following may be the case: One is that children cannot establish a set of alternatives. The other is that although they can establish a set of alternatives, children cannot compute a relevant meaning based on such a set.

The results of an experiment conducted in Terunuma (2004), however, show that neither of them holds. Terunuma (2004) investigates Japanesespeaking children's interpretation of sentences containing the contrastive particle $w a$ 'CTop' such as (33). ${ }^{10}$

(33) Onnanoko-wa koppu-wa arawa-nakat-ta yo. girl-TTop glass-CTop wash-Neg-Past SFP

'The girl didn't wash the glass. (But she washed something other than the glass.)'

In adult Japanese, (33) has a composite meaning of (34a) and (34b).

(34) a. The girl didn't wash the glass.

b. The girl washed something other than the glass.

Like the additive meaning in (26b) above, the contrastive meaning in (34b) is computed based on a set of alternatives.

In Terunuma's (2004) experiment, which used the TVJT methodology, sentences like (33) were judged against two pictures. One picture depicted the context in which a composite meaning like $(34 \mathrm{a}, \mathrm{b})$ was true (the contrastive context). The other depicted the context in which only a meaning like (34a) was true (the neutral context). Seventeen Japanese-speaking children $(4 ; 2-5 ; 1)$ tended to accept sentences like (33) in contrastive contexts but reject them in neutral contexts. These results show that children tend to assign a composite meaning like (34a, b) to sentences like (33).

The results of Terunuma's (2004) experiment indicate that children as

10 The particle $w a$ in Japanese functions as either marking a theme (thematic wa 'TTop') or marking a contrasted element (contrastive wa 'CTop') (Kuno (1973)). As discussed in Kuno (1973), Nakanishi (2007) and McGloin (1987), the two types of wa differ prosodically and syntactically. 
young as the younger children who were tested in Bergsma's experiment can establish a set of alternatives and compute a contrastive meaning based on such a set. This lends some support to Nederstigt's claim that children have the knowledge needed to compute a particular meaning based on a set of alternatives. ${ }^{11,12}$

\section{Concluding Remarks}

I have focused on two topics that are concerned with the acquisition of the linguistic phenomena pertaining to the interface between syntax and semantics: (i) children's scope construal of negative sentences containing

11 The acquisition of particles that require a set of alternatives to be established is also experimentally investigated and discussed in Crain et al. (1992) and Matsuoka (2004). Crain et al. (1992) deal with the English restrictive particle only and Matsuoka (2004) deals with the Japanese additive particle mo 'also.' These studies address the problem of how and why children differ from adults in the way of determining the domain of application for the particles. The discussion in these studies is based on the implicit assumption that children are sensitive to the meaning that is computed based on a set of alternatives, though it is not explicitly shown in their experiment that this is the case.

Paterson et al. (2003), on the other hand, conducted experiments to investigate whether English-speaking children could compute a restrictive meaning based on a set of alternatives in the interpretation of sentences containing only. The results show that 4- to 7 -year-old children often fail to compute such a meaning. However, taking into account that children sometimes assign adult-like interpretation to sentences containing only, Paterson et al. (2003) conclude that they do not lack the knowledge responsible for a restrictive meaning contributed by only.

Paterson et al. (2003) attribute children's failure to compute a meaning based on a set of alternatives to their less well-developed pragmatic knowledge. The question remains as to whether Paterson et al.'s (2003) pragmatic explanation can account for the results of Bergsma's experiment. I leave this question open.

${ }^{12}$ Ito (2008) investigates a particle different from additive, contrastive and restrictive ones. She examines Japanese-speaking children's interpretation of sentences containing sae 'even' such as (i).

(i) Bill-sae Mary-o kiniitteiru.

Bill-even Mary-Acc likes

'Even Bill likes Mary.'

Like additive particles, sae 'even' contributes an additive meaning to sentences. (i), for example, implies that someone other than Bill likes Mary. This additive meaning is computed based on a set of alternatives that consists of Bill and other individuals. Sae 'even' further induces a certain implicature concerning members in a set of alternatives. In (i), the implicature is that Bill is less likely to like Mary than any other members of a set of alternatives. Ito (2008) argues that children fail to compute such implicatures. The problem remains as to why children cannot compute such implicatures. 
a quantifier and (ii) children's knowledge of particles that require a set of alternatives to be established. With respect to (i), Musolino's paper in the book under review claims that children strongly prefer the reading in which the relative scope of a quantifier and negation is isomorphic to their overt c-command relation, and that the source of the isomorphism effect is children's insufficient processing ability. I have pointed out that the findings concerning Japanese-speaking children's interpretation of negative sentences containing zenbu 'all' or $n i$ 'two' in post-Acc position and English-speaking children's interpretation of negative sentences containing every in object position remain to be explained in Musolino. I have also presented a new analysis of children's interpretation of negative sentences containing a quantifier, which leads to the conclusion that the relative scope of a quantifier and negation is isomorphic to their linear order in child language. With respect to (ii), Nederstigt's paper and Bergsma's paper make different claims as to whether children can compute a particular meaning based on a set of alternatives. I have shown that the finding concerning Japanese-speaking children's interpretation of sentences containing a contrastive particle provides some support to Nederstigt's claim that children have the knowledge responsible for establishing a set of alternatives and computing a particular meaning based on such a set.

\section{REFERENCES}

Antinucci, Francesco and Ruth Miller (1976) "How Children Talk about What Happened," Journal of Child Language 3, 167-189.

Berman, Ruth (1985) "The Acquisition of Hebrew," The Crosslinguistic Study of Language Acquisition, Volume I, ed. by Dan I. Slobin, 255-371, Lawrence Erlbaum Associates, Hillsdale, NJ.

Bloom, Lois, Karin Lifter and Jeremie Hafitz (1980) "Semantics of Verbs and the Development of Verb Inflection in Child Language," Language 56, 386-412.

Crain, Stephen, William Philip, Kenneth F. Drozd, Tom Roeper and Kazumi Matsuoka (1992) "Only in Child Language," ms., University of Connecticut.

Crain, Stephen, Weijia Ni and Laura Conway (1994) "Learning, Parsing, and Modularity," Perspectives on Sentence Processing, ed. by Charles Clifton, Jr., Lyn Frazier and Keith Rayner, 443-467, Lawrence Erlbaum Associates, Hillsdale, NJ.

Crain, Stephen and Rosalind Thornton (1998) Investigations in Universal Grammar: A Guide to Experiments on the Acquisition of Syntax and Semantics, MIT Press, Cambridge, MA.

Crain, Stephen, Rosalind Thornton, Carole Boster, Laura Conway, Diane Lillo-Martin 
and Elaine Woodams (1996) "Quantification without Qualification," Language Acquisition 5, 83-153.

Drozd, Kenneth F. (2001) “Children's Weak Interpretations of Universally Quantified Questions," Language Acquisition and Conceptual Development, ed. by Melissa Bowerman and Stephen C. Levinson, 340-376, Cambridge University Press, Cambridge.

Drozd, Kenneth F. and Erik van Loosbroek (1999) "Weak Quantification, Plausible Dissent, and the Development of Children's Pragmatic Competence," Proceedings of the 23rd Boston University Conference on Language Development, 184-195.

Gualmini, Andrea (2003) The Ups and Downs of Child Language: Experimental Studies on Children's Knowledge of Entailment Relationships and Polarity Phenomena, Doctoral dissertation, University of Maryland. [Published by Garland, New York, 2005.]

Gualmini, Andrea, Sarah Hulsey, Valentine Hacquard and Danny Fox (2008) "The Question-Answer Requirement for Scope Assignment," Natural Language Semantics 16, 205-237.

Heim, Irene (1982) The Semantics of Definite and Indefinite Noun Phrases, Doctoral dissertation, University of Massachusetts, Amherst. [Published by Garland, New York, 1988.]

Hornstein, Norbert (1984) Logic as Grammar, MIT Press, Cambridge, MA.

Hornstein, Norbert (1995) Logical Form: From GB to Minimalism, Blackwell, Oxford.

Hulsey, Sarah, Valentine Hacquard, Danny Fox and Andrea Gualmini (2004) "The Question-Answer Requirement and Scope Assignment," MIT Working Papers in Linguistics 48, 71-90.

Ito, Masuyo (2008) “Japanese-speaking Children's Interpretation of the Focus Particle 'Even' Reveals Their (In)sensitivity to Implicatures," An Enterprise in the Cognitive Science of Language: A Festschrift for Yukio Otsu, ed. by Tetsuya Sano, Mika Endo, Miwa Isobe, Koichi Otaki, Koji Sugisaki and Takeru Suzuki, 495-509, Hituzi Shobo, Tokyo.

Kamio, Akio (1983) "Meishiku no Koozoo (The Structure of Noun Phrases)," Nihongo no Kihon Koozoo (The Basic Structure of Japanese), ed. by Kazuko Inoue, 77-126, Sanseido, Tokyo.

Krämer, Irene (2000) Interpreting Indefinites: An Experimental Study of Children's Language Comprehension, Doctoral dissertation, University of Utrecht.

Kuno, Susumu (1973) The Structure of the Japanese Language, MIT Press, Cambridge, MA.

Lidz, Jeffrey and Julien Musolino (2002) "Children's Command of Quantification," Cognition 84, 113-154.

Matsuoka, Kazumi (2004) "Addressing the Syntax/Semantics/Pragmatics Interface: The Acquisition of the Japanese Additive Particle Mo," Online Supplement to the Proceedings of the 28th Boston University Conference on Language Development. <http://128.197.86.186/posters/matsuoka.pdf > 
May, Robert (1985) Logical Form: Its Structure and Derivation, MIT Press, Cambridge, MA.

McGloin, Naomi Hanaoka (1987) "The Role of Wa in Negation," Perspectives on Topicalization: The Case of Japanese Wa," ed. by John Hinds, Senko K. Maynard and Shoichi Iwasaki, 165-183, John Benjamins, Amsterdam.

Moscati, Vincenzo and Andrea Gualmini (to appear) "More Facts that Isomorphism Cannot Explain," Proceedings of the 17th Semantics and Linguistic Theory.

Musolino, Julien (1998) Universal Grammar and the Acquisition of Semantic Knowledge: An Experimental Investigation into the Acquisition of Quantifier-Negation Interaction in English, Doctoral dissertation, University of Maryland.

Musolino, Julien and Jeffrey Lidz (2003) "The Scope Isomorphism: Turning Adults into Children," Language Acquisition 11, 277-291.

Musolino, Julien and Jeffrey Lidz (2006) "Why Children Aren’t Universally Successful with Quantification," Linguistics 44, 817-852.

Nakanishi, Kimiko (2007) "Prosody and Scope Interpretations of the Topic Marker Wa in Japanese," Topic and Focus: Cross-linguistic Perspectives on Meaning and Intonation, ed. by Chungmin Lee, Matthew Gordon and Daniel Büring, 177-193, Springer, Dordrecht.

Paterson, Kevin B., Simon P. Liversedge, Caroline Rowland and Ruth Filik (2003) "Children's Comprehension of Sentences with Focus Particles," Cognition 89, 263-294.

Philip, William (1995) Event Quantification in the Acquisition of Universal Quantification, Doctoral dissertation, University of Massachusetts, Amherst.

Reinhart, Tanya (1997) "Quantifier Scope: How Labor is Divided between QR and Choice Functions," Linguistics and Philosophy 20, 335-397.

Su, Yi-ching (2001) Scope and Specificity in Child Language: A Cross-linguistic Study on English and Chinese, Doctoral dissertation, University of Maryland.

Terunuma, Akiko (2004) "The Acquisition of Contrastive Implicatures," Linguistic Research 20, 155-171, The University of Tokyo English Linguistics Association, Tokyo.

Terunuma, Akiko (2008) “Children's Scope Construal in Negative Sentences Containing a Quantifier," POETICA 70, 39-74.

Watanabe, Akira (2006) "Functional Projections of Nominals in Japanese: Syntax of Classifiers," Natural Language and Linguistic Theory 24, 241-306.

[received April 20 2008, revised and accepted September 20 2008]

Department of English and American Literature

Daito Bunka University

560 Iwadono, Higashi-Matsuyama-shi

Saitama 355-8501

e-mail: terunuma@ic.daito.ac.jp 